\title{
EL DOCTORADO EN EDUCACIÓN: UN EJEMPLO DE DESREGULACIÓN EN EL CAMPO DE LA EDUCACIÓN SUPERIOR EN CHILE*
}

\author{
Javier Corvalán, Alejandra Falabella, María Teresa Rojas ${ }^{1}$
}

\begin{abstract}
RESUMEN
El presente artículo expone los resultados de un estudio sobre el estado de los programas de doctorado en educación en las universidades chilenas, con el objeto de caracterizar su oferta y levantar las principales demandas de los empleadores, como también de los estudiantes de doctorados. El estudio analiza documentos e información en internet, datos secundarios y entrevistas a empleadores, estudiantes y egresados de doctorados en educación. Los resultados obtenidos muestran una amplia oferta de 16 programas doctorales que presentan criterios de admisión, objetivos de formación y núcleos curriculares variados, y con distintos niveles de relevancia y exigencia a la producción y divulgación del conocimiento científico y a su teorización. Junto a esto, los empleadores de doctores en educación y los estudiantes y egresados de los programas expresan múltiples reparos sobre la calidad de la oferta y formación en investigación. Los resultados presentados conforman un ejemplo de la desregulación de la educación superior en Chile y son una muestra de que sus actuales mecanismos regulativos son aún insuficientes, especialmente en relación con estudios de posgrado.
\end{abstract}

Palabras clave: doctorados, posgrado, oferta, regulación

\section{DOCTORAL PROGRAMS IN EDUCATION: AN EXAMPLE OF DEREGULATION IN THE HIGHER EDUCATION FIELD IN CHILE}

\section{ABSTRACT}

This paper presents the results of a study about the situation of the Doctoral Programs in Education in Chile. The study is aimed to characterize the provision of these Doctoral Programs and raise the main demands by employers, as well as doctoral students. It is based upon documents and online information, secondary data and interviews with employers, students and PhD graduates in education. Results show a wide and dispersed supply of 16 doctoral programs with various goals, curriculum plans, and over all, different approaches and levels of demand towards the production and dissemination of scientific knowledge and its theory. Along with this, employers of PhD in education, students and the alumni of programs expressed several critiques about the quality of education provision and research training. The outcomes represent an example of the deregulation of higher education in Chile and indicate that the national current regulatory mechanisms remain inadequate, especially in relation to postgraduate studies.

Keywords: doctorates, post grade, supply, regulation

* El estudio expuesto se realizó en el marco del proyecto "Diseño de Doctorado en Educación" de la Universidad Alberto Hurtado, financiado por el Programa de Mejoramiento de la Calidad de la Educación Superior. Los autores agradecen la colaboración de los consultores Tatiana Cisternas y Daniel Casanova.

1 Investigadores. Facultad de Educación, Universidad Alberto Hurtado, Santiago, Chile. Contacto: jcorvala@cide.cl 


\section{EL DOCTORADO EN EDUCACIÓN: UN EJEMPLO DE DESREGULACIÓN EN EL CAMPO DE LA EDUCACIÓN SUPERIOR EN CHILE}

\section{Introducción}

La educación superior en Chile ha experimentado significativas y complejas transformaciones en las últimas décadas, entremezclando reformas a su estructura y modalidad de financiamiento, nuevas y mayores demandas nacionales e internacionales a la formación terciaria, como también mayores aspiraciones de estudios superiores de parte de los jóvenes y sus familias. La reforma educativa de los años ochenta, de naturaleza liberal, implicó uno de los cambios más radicales en el sistema educativo terciario, desarrollándose un proceso de mercantilización y de desregulación del mismo. Producto de esto se modificó el rol del Estado y su grado de injerencia en este campo, se incorporó la participación de diversos proveedores educativos y se abrió un atractivo nicho de formación educativa y de negocios en el área. Sumado a lo anterior, en los últimos gobiernos se incorporaron nuevos mecanismos de regulación, tales como el sistema nacional de acreditación, y se intensificaron los esfuerzos por aumentar la equidad del acceso a la educación superior. Ello implicó un significativo aumento de la matrícula en educación superior, amparado en créditos estatales y privados.

En este escenario de masificación del ingreso a estudios superiores y de un modelo de educación terciaria escasamente regulado, los estudios de posgrado también se han adaptado a las lógicas de funcionamiento de un sistema universitario mercantil. Entre otras cosas, las regulaciones públicas son débiles en la educación superior chilena y no existen mecanismos para asegurar niveles mínimos de calidad académica de los programas que no están acreditados, en circunstancias que muchos de ellos reciben una cantidad importante de estudiantes cada año.

Teniendo en cuenta esta situación, en este artículo interesa específicamente presentar los resultados de un estudio sobre 
programas de doctorado en educación. Por una parte, se realiza un catastro sobre tales programas en el país y se analiza su oferta a partir de información disponible en internet, una revisión documental y entrevistas realizadas a diez coordinadores o directores de programas. Por otra parte, a partir de 34 entrevistas en profundidad realizadas a potenciales empleadores de doctores en educación, graduados y estudiantes de doctorados, se levantan las demandas que se realizan a estos programas doctorales, graduados y estudiantes de doctorados. De este modo, se intenta contribuir a una comprensión específica sobre la oferta y demanda de este tipo de estudios, como también aportar a una discusión más amplia sobre la (des)regulación de la educación superior chilena, particularmente en estudios de posgrado. El artículo se divide en cinco partes. En primer lugar se expone brevemente un marco conceptual, en segundo lugar se discute sobre el contexto problemático en que surge el estudio, en tercer lugar se exponen los resultados sobre el catastro nacional de los programas doctorales en educación, en cuarto lugar se presentan los resultados sobre las demandas que se le realizan a dichos programas, y finalmente se concluye levantando algunas reflexiones e interrogantes con respecto a la regulación de estudios doctorales en educación.

\section{Marco conceptual del estudio}

El análisis llevado a cabo en este artículo parte del concepto de regulación de los sistemas educacionales de acuerdo a cómo el mismo ha sido operacionalizado en la sociología de la educación europea de las últimas dos décadas (Maroy, 2004). Este concepto refiere a los principios articuladores de los sistemas educativos modernos, los que, debido a su complejidad, requieren elementos de coordinación propuestos, la mayor parte de las veces, por un poder organizador (Dutercq, 2005).

En el caso de los sistemas educativos en general, pero muy especialmente en el caso de la educación superior, los países han constituido -como efecto y como causa de la masificación de tal nivel educacional- una compleja red institucional que, en Chile, es ejemplo de la organización de un mercado propiamente tal. La organización mercantil de la educación superior no está determinada 
solo por aspectos de pago y de precio de determinadas formaciones e instituciones, sino también por la libertad de ofrecer tales formaciones y la posibilidad de escoger entre opciones diversas de las mismas. En el caso de la educación superior chilena, es claro que ella se ha constituido en un mercado abierto ya que cuenta con barreras bajas para la constitución de oferentes, libertad de precio y libre opción de tipo competitivo por parte de los demandantes. Con todo, se trata de un sistema de evidente interés público, a tal punto que el Estado interviene en él con subsidios diversos a las instituciones y/o a los individuos demandantes de ellas.

Si actualmente existe consenso en que todo mercado debe estar sujeto al menos a un nivel mínimo de regulación por parte del Estado, el tema es mucho más evidente en aquellos que -como es el caso de la educación- tiene un evidente interés público y que, además, incorporan recursos del Estado en su funcionamiento. La regulación en este caso busca, por un lado, evitar la desorientación del sistema, es decir, que éste tenga consecuencias distintas e incluso contradictorias a aquellas que la sociedad y el mandato público esperan de él. Por otro lado, la regulación de la educación superior busca ayudar a que los individuos demandantes de formaciones en este nivel tomen las "decisiones correctas", entregándoles mayor información.

Considerando estos principios de análisis, el artículo plantea que la educación superior chilena de posgrado, y en el caso particular del doctorado en educación, está regulada de manera laxa, lo que significa que no existe información suficiente que dé cuenta de la calidad de la oferta y que el principal elemento regulador del Estado chileno -la acreditación- tiene un escaso efecto en orientar tanto la oferta institucional como a los demandantes de tales estudios.

\section{Escenario actual de la educación superior en Chile}

La educación superior chilena fue parte del proceso de transformaciones estructurales del sistema educacional llevado a cabo a comienzo de los años 80 (Bernasconi y Rojas, 2004). Este proceso consistió en una disminución del aporte del Estado al financiamiento de las universidades y en la reestructuración de casas de estudio que tenían 
su sede principal en Santiago (en especial la Universidad de Chile y en particular la Universidad Técnica del Estado que en esa década se rebautizó como Universidad de Santiago), constituyéndose sus sedes regionales en nuevas universidades. Un primer gran movimiento de la reforma de los años 80 en educación superior fue, entonces, financiero y desconcentrador.

Un segundo movimiento fue de tipo privatizador. Consistió en el incentivo a la creación de universidades privadas sin ningún tipo de aporte financiero estatal y con baja regulación del Estado, particularmente en los requisitos académicos de ingreso de sus alumnos. En las tres décadas siguientes, el sistema tuvo otros movimientos que pueden ser leídos en términos de mayor o menor por parte del Estado y en los cuales se plasmaron políticas reguladoras y expansivas de la educación superior provenientes de los gobiernos de la Concertación. Estas regulaciones se refieren a dos ámbitos: i) la calidad de las instituciones y de los programas (establecimiento de un sistema de acreditación); y ii) los requisitos para recibir financiamientos del Estado, suplementarios al Aporte Fiscal Directo (AFD), en el caso de las universidades tradicionales, y excepcionales en el caso de las universidades privadas, basados en el crédito a los alumnos.

Pasados casi 30 años del inicio de estas reformas el panorama es ya bastante conocido: poco más de la mitad de los alumnos de pregrado estudian en las universidades privadas no tradicionales (54\% en el año 2009²), un grupo pequeño de universidades tradicionales se mantienen altamente selectivas (Brunner y Uribe, 2007), las actividades de investigación se concentran casi exclusivamente en las universidades tradicionales y en muy pocas privadas ${ }^{3}$, en tanto la oferta y el crecimiento de matrícula de las universidades privadas se concentra en pocas carreras que, por lo general, requieren baja inversión. Otro aspecto de gran importancia para el análisis de las dinámicas de la educación superior en los últimos años ha sido su masificación, la que se ha concretado por la vía de una democratización

2 Fuente: www.divesup.cl, revisado el 2 de noviembre de 2010.

3 Por ejemplo en el concurso FONDECYT regular del año 2007, el 67\% de los proyectos aprobados se concentró en cinco universidades, todas del Consejo de Rectores. 
del ingreso. Así, mientras el año 1990 la cobertura sumada de los tres primeros quintiles era en el pregrado del orden de un $11 \%$, en el año 2009 la misma llega al 52\%4.

A partir de la segunda mitad de los años 90 y con particular fuerza en la década del 2000, el campo universitario chileno agrega como tema central a su conversación un elemento hasta entonces escasamente presente: el posgrado ${ }^{5}$. En efecto, a pesar de que existen universidades chilenas con más de un siglo de vida y a pesar de que el mundo universitario chileno tiene niveles importantes de consolidación desde hace al menos medio siglo, la presencia de estudios de posgrado en ellas ha sido particularmente baja y concentrada en áreas de ciencias básicas (referido al doctorado, ver Krauskopf, 1999).

La proliferación de las formaciones de posgrado, particularmente en áreas de ciencias sociales y de educación, es algo que ha ocurrido también en otros países de América Latina en el mismo periodo y que tiene diversas explicaciones socioeducativas. La primera de ellas se refiere a una devaluación educativa producto de la masificación de las formaciones de pregrado. Este tema ha sido estudiado por la sociología de la educación a propósito de la masificación de la educación escolar lo que, entre otros elementos, ha aumentado la presión por ingresar a formaciones superiores. De esta manera, el mercado laboral pone mayores exigencias para el desempeño de una misma labor, y por medio de la proliferación de diplomas se llega a una sociedad "credencialista" (Collins, 1989). Visto de esta manera, este parece ser un asunto solamente de forma y no de fondo, ya que se supone que se exigen mayores calificaciones para un trabajo que, en realidad, requiere de menores aptitudes. En contrapartida se señala el argumento de mayor productividad, a partir de la misma labor, en

4 Fuente: www.casen.cl, revisado 30 de octubre de 2010.

5 Ejemplo de esto es que en el periodo 2002-2008 en las universidades del Consejo de Rectores la matrícula de pregrado ha crecido un $18,5 \%$ mientras que la de magíster lo ha hecho un 109\% y la de doctorado un 102\% (fuente www.cruch.cl, revisado el 30 de septiembre de 2010). Considerando la totalidad del sistema universitario el aumento de matrícula de magíster creció solo un 3,6\% en el periodo 1983-1990, un 248\% entre 1990 y 2000 y un $257 \%$ en el periodo $2000-2009$. En el doctorado las cifras de aumento son de 148\% (1983-1990), de 337\% (1990-2000) y de un 254\% (2000-2009) (fuente www.divesup.cl. Revisado 30 de septiembre de 2010). 
individuos que tienen mayor calificación y certificación (argumento retomado por la teoría del capital humano, ver Becker, 1966). Se vuelve aquí a un antiguo argumento: lo importante no es lo que se hace, sino más bien quién lo hace.

Por otro lado, parece indudable que en el caso chileno el posgrado también es una consecuencia de factores como las señales y los recursos asignados por el Estado, así como de procesos de distinción generados por las propias universidades. En cuanto a la acción del Estado, a partir de los procesos de acreditación de instituciones iniciados en la década del $2000^{6}$, se estableció la categoría de docencia de posgrado generando una distinción entre las instituciones que la poseen y aquellas que no la tienen ${ }^{7}$. Paralelamente, es necesario señalar que en Chile no existe hasta el momento una ley que regule las formaciones de posgrado, por lo cual ellas pueden existir bajo cualquier estructura curricular, admitiendo cualquier formación previa y teniendo distintas duraciones. Desde este punto de vista, el sistema de posgrado chileno está fuertemente desregulado y la acreditación intenta actuar como poder regulador.

\section{La oferta de doctorados en educación en Chile}

En esta sección interesa exponer algunos de los resultados de un catastro que analizó la oferta de los programas de doctorados en educación en dieciséis universidades chilenas. El propósito de ello es levantar información sobre la oferta de tales programas doctorales, su matrícula, mecanismos de selección, número de graduados y planes de estudio (duración, objetivos, cursos, trayectoria de la tesis). Para este fin se analizó la información disponible en las páginas web de universidades, la documentación y folletería que entrega cada programa, y datos secundarios del Sistema Nacional de Información para la Educación Superior y de la Comisión Nacional de Acreditación. Sumado a ello, se realizaron diez entrevistas a informantes claves para corroborar o complementar la información. Es importante relevar que no siempre se pudo acceder a información sobre todos

6 Ver www.cnachile.cl, revisado 10 de octubre de 2010.

7 En 2010 solo 7 universidades están acreditadas en esa área (docencia de posgrado). Fuente: www.cnachile.cl, revisado 10 de octubre de 2010. 
los aspectos mencionados en cada uno de los programas debido a la escasa disponibilidad pública que existe de la misma, razón por la cual hay cierta variación en el número total de programas estudiados en los análisis estadísticos que se realizan a continuación.

\section{a) Oferta de programas de doctorado en educación}

Desde fines de los 80 y hasta la década del 90 solo hubo dos ofertas de doctorado en educación en el país, una en la Pontificia Universidad Católica y otra en la Universidad Academia de Humanismo Cristiano ${ }^{8}$. Sin embargo, en los últimos años, especialmente desde el 2005, ha habido una explosión de estos programas, lo que ha implicado un significativo crecimiento en la matrícula. En uno de los estudios que sirve de base a este artículo se observa que, durante el año 2009 había 16 programas $^{9}$ en 14 instituciones (tabla n. $\left.{ }^{\circ} 1\right)^{10}$.

Tabla n. ${ }^{\circ}$ 1: Doctorados en Educación nacionales

\begin{tabular}{|c|l|l|l|}
\hline $\mathrm{N}^{0}$ & INSTITUCIÓN & PROGRAMA & SEDE \\
\hline 1. & $\begin{array}{l}\text { Universidad Academia Humanismo } \\
\text { Cristiano }\end{array}$ & Doctorado en Educación & Santiago \\
\hline 2. & Universidad de Aconcagua & $\begin{array}{l}\text { Doctorado en Educación, mención } \\
\text { en Administración y Gestión de la } \\
\text { Educación }\end{array}$ & Talca \\
\hline 3. & Universidad de Aconcagua & $\begin{array}{l}\text { Doctorado en Educación, mención } \\
\text { en Evaluación y Acreditación }\end{array}$ & Talca \\
\hline 4. & Universidad de Aconcagua & $\begin{array}{l}\text { Doctorado en Educación, mención } \\
\text { en Curriculum y Didáctica }\end{array}$ & Talca \\
\hline 5. & $\begin{array}{l}\text { Universidad de Artes y Ciencias } \\
\text { Sociales }\end{array}$ & $\begin{array}{l}\text { Doctorado en Cultura y Educación } \\
\text { en América Latina }\end{array}$ & Santiago \\
\hline 6. & $\begin{array}{l}\text { Universidad de Sevilla - Universidad } \\
\text { Autónoma de Chile }\end{array}$ & $\begin{array}{l}\text { Doctorado en Ciencias de la } \\
\text { Educación (didáctica y organización } \\
\text { de instituciones educativas) }\end{array}$ & Santiago \\
\hline 7. & Universidad Bolivariana & $\begin{array}{l}\text { Doctorado en Educación } \\
\text { Aprendizaje Transformacional }\end{array}$ & Santiago \\
\hline
\end{tabular}

8 Ambos doctorados fueron creados el año 1991.

9 También se identificó la oferta de un doctorado en educación de la Universidad de los Lagos, en convenio con la Universidad de Valladolid (España), pero se declaró que este se había cerrado el año 2005.

10 Ello representa más del doble del número de programas doctorales en otras áreas, como por ejemplo en psicología o sociología. Al año 2010 se ofrecen seis programas doctorales en el área de la psicología, dictadas en cuatro universidades; y seis programas doctorales en ciencias sociales, sociología o estudios latinoamericanos. (Fuente: Sistema de Información de Educación Superior, http://www.divesup.cl/sies/). 


\begin{tabular}{|c|l|l|l|}
\hline 8. & $\begin{array}{l}\text { Pontificia Universidad Católica de } \\
\text { Chile }\end{array}$ & $\begin{array}{l}\text { Doctorado en Ciencias de la } \\
\text { Educación }\end{array}$ & Santiago \\
\hline 9. & $\begin{array}{l}\text { Universidad de Granada - } \\
\text { Universidad Central }\end{array}$ & Doctorado en Psicología y Educación & Santiago \\
\hline 10. & $\begin{array}{l}\text { Universidad de Concepción } \\
\text { (convenio con la Universidad de } \\
\text { Estocolmo) }\end{array}$ & Doctorado en Educación & Concepción \\
\hline 11. & Universidad de La Frontera* & $\begin{array}{l}\text { Doctorado en Ciencias de la } \\
\text { Educación }\end{array}$ & Temuco \\
\hline 12. & $\begin{array}{l}\text { Universidad de Granada - } \\
\text { Universidad } \\
\text { del Mar }\end{array}$ & $\begin{array}{l}\text { Doctorado en Educación. } \\
\text { Curriculum, Profesorado e } \\
\text { Instituciones Educativas }\end{array}$ & Viña del Mar \\
\hline 13. & $\begin{array}{l}\text { Universidad Metropolitana de las } \\
\text { Ciencias de la Educación }\end{array}$ & Doctorado en Educación & Santiago \\
\hline 14. & $\begin{array}{l}\text { Universidad de Playa Ancha } \\
\text { Doctorado en Políticas y Gestión } \\
\text { Educacional }\end{array}$ & Valparaíso \\
\hline 15. & $\begin{array}{l}\text { Universidad del País Vasco - } \\
\text { Universidad Arturo Prat }\end{array}$ & Doctorado en Psicología y Educación & Iquique \\
\hline 16. & $\begin{array}{l}\text { Universidad Nacional de Educación } \\
\text { Distancia (UnED), de España - } \\
\text { Universidad Mayor }\end{array}$ & Doctorado en Educación & $\begin{array}{l}\text { Temuco / } \\
\text { Santiago }\end{array}$ \\
\hline
\end{tabular}

De los doctorados catastrados, cuatro se declararon sin ingreso de estudiantes nuevos, aun cuando se reconocían en actividad con alumnos ingresados los años anteriores. Se trata de los programas ofrecidos por las universidades Central, de Concepción, del Mar y Mayor. Esta situación, según lo declarado, ocurre por distintos motivos, tales como cierre de convenios con universidades extranjeras, evaluación interna al programa y/o falta de planta académica para guiar tesis. Por otra parte, cabe destacar que existe un modelo de programa que consiste en que una universidad extranjera ofrece un doctorado y otorga el título con sede en una universidad nacional. Este es el caso de los programas ofrecidos en las universidades Autónoma de Chile, Central, Del Mar y Mayor.

Es relevante también mencionar que el año 2010 se crearon dos nuevos doctorados: el Doctorado en Educación Superior de la Universidad Diego Portales, en conjunto con la Universidad de Leiden (Holanda), y el Doctorado en Ciencias de la Educación, con

* Esta universidad también creó el Doctorado en Ciencias de la Educación mención en Evaluación Educacional, sin embargo, este programa finalmente no se ejecutó por falta de postulantes. 
mención en educación intercultural de la Universidad de Santiago de Chile. Dado que este artículo se basa en información recopilada el año 2009, estos dos últimos programas no están incluidos en los casos estudiados ni en el análisis global.

En consistencia con la amplia oferta de programas de doctorado en educación, la matrícula nacional el año 2009 es de al menos 524 estudiantes, número que debiera aumentar, considerando que no hay información disponible de seis programas (ver tabla n. ${ }^{\circ}$ 2). El promedio de edad de esta matrícula es de 45 años de edad, con un $57 \%$ de hombres y $43 \%$ de mujeres, en tanto el número de titulación anual en estos programas, de acuerdo a la información pública de las universidades del Consejo de Rectores ${ }^{11}$, es de 10 doctores anuales en promedio entre los años 2004 a 2009, sobre un ingreso medio anual de 30 estudiantes.

Tabla n. ${ }^{\circ}$ 2. Matrícula por institución y sexo, al año 2009

\begin{tabular}{|l|c|c|c|}
\hline INSTITUCIÓN & MUJERES & HOMBRES & TOTAL \\
\hline Universidad Academia Humanismo Cristiano & 9 & 7 & 16 \\
\hline Universidad de Aconcagua & 115 & 193 & 308 \\
\hline Universidad de Artes y Ciencias Sociales & 7 & 8 & 15 \\
\hline Pontificia Universidad Católica de Chile & 38 & 20 & 58 \\
\hline Universidad Bolivariana & 35 & 36 & 71 \\
\hline Universidad de Concepción & 5 & 2 & 7 \\
\hline Universidad Metropolitana de Ciencias de la Educación & 9 & 21 & 30 \\
\hline Universidad de Playa Ancha & 9 & 10 & 19 \\
\hline TOTAL & 227 & 297 & 524 \\
\hline
\end{tabular}

Fuente: elaboración a partir de Sistema Nacional de Información para la Educación Superior (SIES) y consulta a instituciones.

De todos los programas de doctorado ofertados hasta el 2009, solo dos han postulado a la acreditación y solo uno había sido acreditado (el de la Pontificia Universidad Católica de Chile), por tanto el grueso de la matrícula estudia en programas no acreditados, en los cuales no es posible acceder a becas de Estado.

11 Las universidades del CRUCH que ofrecen doctorados en educación son: Pontificia Universidad Católica de Chile, De Concepción, Metropolitana de las Ciencias de la Educación, de Playa Ancha y Arturo Prat. 


\section{b) Programa de estudios}

Los resultados del estudio de los programas muestran una oferta heterogénea y dispersa, con distintos niveles de exigencia, duración y cursos temáticos en los que, además, se otorga desiguales grados de relevancia a la formación en investigación. A continuación se expondrán los principales hallazgos del análisis realizado a este respecto.

i) Requisitos de ingreso: En cuanto a los requisitos de ingreso, se evidencia que existen variados niveles de exigencia de entrada a los programas de doctorado. El único requisito que todos los programas exigen por igual es la licenciatura o título equivalente. Sobre la base del análisis realizado se identificaron tres niveles de exigencias para el ingreso al doctorado. Un primer nivel es el de programas con un alto grado de selectividad, el cual busca estudiantes con un perfil de académico-investigador. Además de solicitar la licenciatura o título de magíster, estos programas exigen presentar un proyecto de investigación y manejar un segundo idioma, como también otros requisitos, tales como la aprobación de un examen, cartas de recomendación, experiencia en investigación y, en un caso, una producción escrita, como un artículo publicado o un capítulo de una tesis. En este nivel se encuentran solamente tres programas doctorales, lo cual representa el $25 \%$ de los programas analizados.

En el nivel medio de selección se identifican otros tres programas doctorales (25\%) los cuales, además de exigir la licenciatura o un título equivalente, exigen otros antecedentes, como una carta de intenciones (referido al tema de tesis) o cartas de recomendación, pero no exigen, por ejemplo, manejo de un segundo idioma o la entrega de una producción escrita. En el nivel bajo se ubica el 50\% restante de los programas doctorales, que no exige ningún otro antecedente aparte de la licenciatura o título equivalente.

Tabla n. ${ }^{\circ}$ 3: Selección de los estudiantes

\begin{tabular}{|l|c|c|}
\hline Requisitos & $\mathrm{N}^{0}$ de programas & Porcentaje \\
\hline Alto nivel de selección & 3 & $25 \%$ \\
\hline Medio nivel de selección & 3 & $25 \%$ \\
\hline Bajo nivel de selección & 6 & $50 \%$ \\
\hline Total & 12 & $100 \%$ \\
\hline
\end{tabular}

* Cuatro programas doctorales sin información. 
Estos resultados indican que por lo general se observa un bajo nivel de filtro en la selección de los estudiantes doctorales. Solamente tres programas, de acuerdo a la información entregada, indican un conjunto de exigencias que refieren a una mayor selectividad de entrada a los estudiantes.

ii) Duración y modalidad de enseñanza: La duración total declarada de los estudios varía de cinco a diez semestres considerados hasta la obtención efectiva del grado, es decir, varía en hasta un $100 \%$, mientras el promedio del tiempo de los programas es de siete semestres. En relación a las modalidades de enseñanza, seis programas ofrecen una modalidad presencial, seis una de tipo semipresencial y dos se llevan a cabo a distancia. Esto significa que alrededor de un $43 \%$ de los programas tiene una modalidad presencial y $57 \%$ tiene una modalidad a distancia o semipresencial. Solamente dos programas doctorales establecen exigencias explícitas en cuanto a las jornadas de trabajo de los estudiantes, uno de ellos exige dedicación completa los dos primeros años y el otro una disposición de media jornada (22 horas semanales).

iii) Propósitos de formación: Los propósitos generales de los programas de estudio, en su mayoría, están vinculados a la formación de investigadores en el campo de la educación. No obstante, la relevancia y centralidad que se le otorga al desarrollo de competencias para la investigación oscila de forma significativa entre los programas. Además, se identificaron tres tipos de planes de estudio que representan diferentes aproximaciones a la investigación: i) académico, ii) pragmático, iii) experiencial. El primero está centrado en la formación de competencias en investigación, otorgándosele prioridad a la inserción del estudiante en una comunidad de investigación, a la enseñanza de metodologías de investigación, y a la producción y comunicación de conocimiento científico. A continuación se presenta, a modo de ejemplo, el propósito general de uno de estos programas:

"Tiene como propósito fomentar el desarrollo de las ciencias de la educación y formar doctores que contribuyan al desarrollo de la investigación y a la 
comunicación del conocimiento científico en educación. La Facultad de Educación sustenta la idea de que, la formación de un doctor en Ciencias de la Educación se hace en el proceso mismo de la generación del conocimiento. Un investigador se forma investigando. Por tanto, el énfasis central o la columna vertebral del proceso formativo es la participación del candidato inserto en las condiciones reales en que se da el proceso, vinculado a un equipo académico del mejor nivel y de productividad."

En este primer tipo se identificaron seis programas doctorales. Estos programas de doctorados varían en el campo de estudio, pues algunos están situados desde el campo de la pedagogía y/o didáctica, otros desde las ciencias de la educación en genérico, y otro tiene particular énfasis en la investigación desde las ciencias sociales. Sin embargo, lo común a estos programas es que buscan producir investigación de altos estándares de rigor, que contribuya a la producción de conocimiento empírico-teórico. Consistentemente con este enfoque, el perfil de egreso de estos programas está centrado en formar profesionales investigadores capaces de gestionar proyectos de investigación, producir conocimiento científico y divulgar sus resultados.

En contrapartida, se identificó un programa de doctorado que le otorga un enfoque más instrumental y pragmático a la investigación. Esta formación entrega herramientas de investigación y actualización de conocimientos teóricos, no obstante el propósito de su formación está centrado en la evaluación y mejoramiento de la práctica educativa, donde la teorización del fenómeno educativo adquiere un rol secundario. El perfil de los estudiantes de estos programas se relaciona centralmente con profesionales que trabajan en el área de evaluación, asesoría e intervención educativa, como también pueden estar vinculados a la docencia universitaria. El análisis arrojó que seis programas se encuentran en este tipo (46\%), al cual caracteriza la siguiente cita:

"El doctorado [...] tiene como propósito formar profesionales con amplio y profundo dominio en las diversas metodologías de la investigación, entregando 
principios, criterios y fundamentos que les permitan el diseño desarrollo y gestión de políticas educativas innovadoras en los niveles básico, medio y superior, tanto en el sector público como privado, generando alternativas de soluciones efectivas a los problemas de desarrollo educativo y cultural."

Por último, se identificó un tercer tipo en que se ubica un programa de estudio, el cual se ejemplifica en la siguiente cita:

"Este espacio doctoral promueve el desenvolvimiento de disposiciones emocionales, racionales y corporales que favorezcan la emergencia de conversaciones creativas, donde sostener procesos de aprendizaje y transformación profunda. Adoptando esta premisa del pensamiento complejo (oscilación dinámica), propone su desenvolvimiento en la realidad como campo, recreando la forma y el espacio en que se manifiesta."

Aunque este programa de estudio incorpora trabajos de investigación, no ubica la formación en investigación como un eje central en el plan de estudios, sino que más bien la indagación y reflexión tanto individual como colectiva, en vinculación con las experiencias personales de cada estudiante. Además, se enfatiza el desarrollo y actualización de una aproximación teórica específica en el campo de la educación.

Tabla n. ${ }^{\circ}$ 4: Síntesis de propósitos de formación

\begin{tabular}{|l|l|c|c|}
\hline Tipo & Propósito central & $\mathrm{N}^{0}$ programas & Porcentaje \\
\hline Académico & $\begin{array}{l}\text { Formación en competencias en investigación } \\
\text { para la producción, teorización y divulgación del } \\
\text { conocimiento científico }\end{array}$ & 6 & $46 \%$ \\
\hline Pragmático & $\begin{array}{l}\text { Entrega de herramientas de investigación para la } \\
\text { evaluación, intervención y mejoramiento de la } \\
\text { práctica pedagógica }\end{array}$ & 6 & $46 \%$ \\
\hline Experiencial & $\begin{array}{l}\text { Indagación y reflexión sobre paradigmas } \\
\text { educativos, en vinculación con las experiencias } \\
\text { personales y colectivas. }\end{array}$ & 1 & $8 \%$ \\
\hline
\end{tabular}

* Tres programas doctorales sin información.

Estos tipos se asocian a distintos enfoques epistemológicos dentro de la investigación en educación, como son el científico, el 
pragmático y el experiencial, ${ }^{12}$ los cuales involucran diferentes fines, aproximaciones metodológicas, criterios de rigor y mecanismos de validación. Estas diferencias en la formación doctoral abren interrogantes sobre la distinción de un doctorado con otros estudios, como el grado de magíster. A su vez, permite reflexionar sobre si estos disímiles enfoques de investigación expuestos tienen cabida en la formación doctoral que representa el grado académico máximo en investigación o si se requieren nuevas distinciones en los títulos otorgados entre un doctorado de enfoque académico-científico y uno de investigación profesionalizante. Esta distinción existe, por ejemplo, en el mundo anglosajón entre el Doctor of Philosohy in Education (PhD) y el Doctorate in Education (EdD).

iv) Estructura de los planes de estudio: Es posible identificar algunas tendencias generales en cuanto a la estructura de los planes de estudio. La mayoría de los programas ofrecen cursos durante los primeros dos a cuatro semestres. Luego, se presenta un proyecto de tesis, un examen de suficiencia y/o candidatura, para finalizar con un examen de grado. No se encontró evidencia de normativas detalladas del proceso de tesis (como existen en doctorados extranjeros). Una distinción que se halló en tres de los programas (dos asociados a universidades españolas) es una etapa intermedia dentro del programa doctoral, en que luego de la aprobación de un conjunto de cursos y de un proyecto de investigación o tesina, se entrega un título con grado de magíster.

v) Cursos de los planes de estudio: Un análisis de la distribución porcentual de ocho planes de estudios disponibles muestra que la mayor parte de los cursos se centra en la metodología de la investigación (38\%) como se observa en la tabla n. ${ }^{\circ}$ 5. Sin embargo, este porcentaje oscila de un $91 \%$ a un $14 \%$ entre los distintos programas, lo cual demuestra nuevamente una variación significativa entre la relevancia que se le otorga a la formación en investigación. Además, llama la atención que, a diferencia de la tendencia internacional, no se encontraron objetivos programáticos, ni cursos o talleres para

12 Para mayor discusión sobre paradigmas en investigación educativa ver por ejemplo: Jimeno Sacristán y Pérez Gómez (2008). 
formar de manera explícita competencias genéricas como escritura académica, discusión bibliográfica, comunicación de resultados de investigación, o postulación a fondos concursables, aunque en dos casos se encontró evidencia de evaluaciones intermedias relacionadas a publicación de artículos y postulación a proyectos.

Tabla n. ${ }^{0} 5$. Distribución porcentual de cursos ${ }^{13}$

\begin{tabular}{|l|c|}
\hline CATEGORÍA & PROMEDIO \\
\hline Metodología de investigación & $38,1 \%$ \\
\hline Estrategias pedagógicas, didáctica, evaluación, currículum & $15,5 \%$ \\
\hline Epistemología y teoría de la educación & $15,1 \%$ \\
\hline Gestión escolar y cambio organizacional & $15,7 \%$ \\
\hline Políticas, sociología y economía de la educación & $10,5 \%$ \\
\hline Teoría y ciencias del aprendizaje (ciencias cognitivas) & $4,7 \%$ \\
\hline Total & $100,0 \%$ \\
\hline
\end{tabular}

En la tabla n. ${ }^{\circ} 5$ se observa que los planes de estudios ponen énfasis en contenidos relacionados a curriculum y pedagogía, epistemología y teoría educativa, como también se ha incorporado el área de estudio de la gestión escolar. En menor medida se identificó contenidos relacionados a política y sociología de la educación, teorías del aprendizaje y trabajo educativo en contextos de vulnerabilidad socio-educativa. Además, el análisis de los planes de estudio evidenció en la mayoría de los casos una alta rigidez de los programas y una escasa oferta de cursos optativos y de cursos interdisciplinarios. No obstante, en dos casos en particular, se observa una interesante amplitud de cursos que se ofrecen en vinculación con otras facultades de la misma universidad.

vi) Áreas de investigación: En cuanto a la oferta de los programas, en su mayoría no se identifican líneas de investigación determinadas, sino que más bien se hace referencia de forma genérica a investigación en el campo de la educación. Por tanto, se infiere que son los intereses de los estudiantes, más o menos negociados e influenciados por el cuerpo académico, los que inclinan las temáticas de investigación. Sin embargo, han surgido en los últimos años

13 Este análisis se realizó sobre la base de información disponible de ocho programas doctorales. 
programas doctorales con menciones más específicas o que explicitan líneas de investigación ${ }^{14}$.

\section{c) Condiciones institucionales}

El estudio evidenció precarias e inestables condiciones institucionales de parte de varios programas doctorales. Por ejemplo, como se mencionó anteriormente solo uno de los programas está acreditado, cuatro han cerrado su matrícula a estudiantes nuevos, y además, en varios programas se ofrece escasa información pública sobre la oferta. Igualmente los coordinadores y directores (nueve de los diez entrevistados) expresan que uno de los mayores problemas de mantener un doctorado es la falta de planta académica estable y de profesores para guiar las tesis doctorales. Es decir, mientras los programas encuentran una alta demanda de estudiantes interesados en matricularse, las instituciones se enfrentan con dificultades para tener un equipo suficiente de doctores que supervisen a los estudiantes. De hecho, solamente seis de los programas entregan información pública de su planta docente. Del mismo modo, se reconoce una escasez de líneas de investigación consolidadas en las cuales los estudiantes puedan insertarse, lo que preocupa especialmente en aquellos programas con un enfoque académicoinvestigativo.

Junto a ello, se observan débiles redes de cooperación académica nacionales e internacionales. En el ámbito nacional llama la atención el grado de dispersión y de aislamiento entre los programas doctorales, no observándose modalidades de colaboración interuniversitaria. En relación con vínculos universitarios internacionales, como se mencionó anteriormente, existen programas que tienen convenios con universidades extranjeras en que son estas últimas las responsables de la formación doctoral. Por lo tanto, no pareciera que en tales casos hubiese colaboración académica, sino más bien un programa preestablecido que es ejecutado por una institución externa, al interior de una universidad nacional. Aparte de estos casos, se identifican solamente dos instituciones en que se

14 Por ejemplo, los programas de las universidades Autónoma de Chile, De Concepción, De La Frontera, y De Playa Ancha. 
declaran sistemas de intercambios académicos institucionalizados como parte de la gestión general de la universidad.

Síntesis del estado de la oferta de doctorados en educación en Chile

- Existe una amplia y diversa oferta de doctorados en educación, con al menos 16 programas con más de 500 estudiantes, al año 2009. Basándose en la evidencia analizada es posible reconocer la tendencia de una oferta curricular heterogénea y dispersa, de variadas duraciones (de cinco a diez semestres), con distintas modalidades de enseñanza (presencial, semipresencial y a distancia), como también con distintos niveles de exigencia para la selección de los estudiantes. De la misma manera, se observan distintos enfoques curriculares y planes de estudio, en que algunos programas dan escasa relevancia a la formación en investigación, tanto en sus objetivos declarados, como en el diseño del plan de estudio y en la presencia de cursos de metodología de investigación. Estos resultados reflejan que no existe un consenso sobre los objetivos, enfoques y niveles de exigencias mínimos de un programa doctoral en educación en Chile y sobre los requisitos y exigencias para cursarlo.

- La oferta de los programas en su mayoría es genérica en el campo de la educación, y se reconocen escasos programas especializados en líneas consolidadas de investigación, en los cuales se puedan insertar los estudiantes (con algunas excepciones). Sumado a ello, se identificaron en ciertos casos precarias condiciones institucionales en cuanto al bajo número de doctores para guiar a los estudiantes en sus tesis doctorales e insertarlos dentro de equipos de investigación. Adicionalmente, no hay evidencia de cooperación académica entre universidades nacionales y se observa un bajo nivel de intercambio con universidades internacionales, lo cual podría eventualmente enmendar debilidades institucionales y potenciar la oferta académica.

- Existen casos de programas doctorales que ofrecen planes consistentes, rigurosos y exigentes, con altos filtros de selección, sumados a condiciones institucionales consolidadas, tanto en investigación como en cuanto a colaboración académica internacional. Paralelamente, se pone en duda la calidad y 
condiciones institucionales de algunos programas, especialmente aquellos que: i) no colocan filtros de entrada a sus estudiantes, ii) en sus planes de estudio no dan relevancia a la formación de competencias académicas en investigación, iii) no cuentan con líneas de investigación y con una planta académica suficiente para guiar a los estudiantes en sus tesis doctorales, iv) no publican información sobre el programa (propósito, plan de estudios, planta docente, modos de evaluación), y v) no están acreditados como institución o programa.

\section{Demandas a los doctorados en educación: visiones de empleadores, graduados y estudiantes de doctorado}

En este apartado se analizan las opiniones de diversos actores vinculados a programas doctorales en educación con la finalidad de levantar un conjunto de demandas específicas a las propuestas formativas de los programas nacionales. En particular, se recogieron opiniones sobre los perfiles académicos esperados en los doctores en educación, de manera de precisar si existe la percepción de que los programas doctorales nacionales logran formar académicos que cumplan con dichos perfiles y, además, se preguntó sobre la percepción de la calidad académica de los programas doctorales nacionales en el área de la educación.

La información recabada es de carácter cualitativo y se obtuvo a través de entrevistas individuales. Conocer las opiniones de actores importantes del campo de los posgrados acerca de la calidad de los programas de doctorados en educación permite identificar, desde otra perspectiva, las posibles debilidades de la oferta de doctorados en educación nacionales. Por ello, nos pareció pertinente consultar a tres actores: los potenciales empleadores de doctores en educación, los graduados y los estudiantes de doctorados chilenos. Entre los primeros, se encuentran principalmente los decanos de facultades de educación, pues es en las universidades donde se concentra la demanda por doctores en educación. Por otra parte, preguntamos a graduados y estudiantes de doctorados nacionales e internacionales, pues, dado que vivieron o están viviendo un proceso de formación 
como investigadores, nos pareció interesante conocer su percepción acerca de la calidad de sus trayectorias académicas.

Tabla n. ${ }^{\circ}$ 6. Actores entrevistados

\begin{tabular}{|c|c|c|}
\hline Empleadores & $\begin{array}{l}\text { Graduados de doctorados en } \\
\text { educación }\end{array}$ & $\begin{array}{l}\text { Estudiantes actuales de } \\
\text { doctorados en educación }\end{array}$ \\
\hline $\begin{array}{l}5 \text { decanos de facultades de } \\
\text { educación de universidades } \\
\text { pertenecientes al Consejo de } \\
\text { Rectores. }\end{array}$ & $\begin{array}{l}4 \text { graduados de doctorado } \\
\text { nacional en educación acreditado. }\end{array}$ & $\begin{array}{l}4 \text { estudiantes cursando } \\
\text { doctorado nacional en } \\
\text { educación acreditados. }\end{array}$ \\
\hline $\begin{array}{l}2 \text { decanos de facultades de } \\
\text { educación de universidades } \\
\text { privadas acreditadas. } \\
1 \text { vicerrector de universidad } \\
\text { privada acreditada. }\end{array}$ & $\begin{array}{l}2 \text { graduados de doctorados } \\
\text { nacionales en educación no } \\
\text { acreditados. }\end{array}$ & $\begin{array}{l}6 \text { estudiantes cursando } \\
\text { doctorados nacionales en } \\
\text { educación no acreditados. }\end{array}$ \\
\hline $\begin{array}{l}3 \text { directores de fundaciones } \\
\text { educacionales }\end{array}$ & \multirow{2}{*}{$\begin{array}{l}2 \text { graduados de doctorado } \\
\text { internacional en educación en } \\
\text { países de habla hispana. }\end{array}$} & \\
\hline $\begin{array}{l}1 \text { representante del Ministerio de } \\
\text { Educación }\end{array}$ & & \\
\hline $\begin{array}{l}1 \text { director de centro de } \\
\text { investigación universitario } \\
1 \text { director de consultora privada }\end{array}$ & \begin{tabular}{|l|}
2 graduados de doctorado \\
internacional en educación en \\
países que requieren el manejo de \\
segunda lengua
\end{tabular} & \\
\hline \multicolumn{3}{|c|}{ Total: 34 entrevistados } \\
\hline
\end{tabular}

Entre las opiniones recogidas, cabe destacar:

\section{a) Perfiles de doctores en educación requeridos por los empleadores}

Las universidades son las principales demandantes de doctores en educación. Al contrario, las instituciones públicas, las fundaciones y las consultoras no demandan directamente doctores, sino profesionales con habilidades específicas que pueden coincidir o no con profesionales con el grado de doctor. Respecto a las universidades, los entrevistados son claros en enfatizar que el prestigio de las facultades se juega hoy día por la cantidad de doctores que posea:

"Mirado desde las políticas públicas, tener hartos doctores implica reconocimiento. También, mientras más doctores tengamos también hay más posibilidad de apoyo económico" (decano de una facultad de Educación, universidad del Consejo de Rectores). 
Las autoridades universitarias consideran que el perfil ideal de un doctor en educación es un académico con experiencia en investigación relevante, conocimiento en profundidad del sistema educativo chileno, con un número considerable de publicaciones y asistencia a congresos y con experticia en docencia universitaria. Sin embargo, consideran que ser graduado de un doctorado no garantiza sensibilidad o experticia en la docencia universitaria, cuestión particularmente importante en las facultades que forman profesores. Según los entrevistados, este perfil es muy difícil de encontrar en el contexto nacional. La experiencia les indica que los programas doctorales nacionales suelen ser débiles en formación en investigación, especialmente en el ámbito de los estudios cuantitativos.

A pesar de la valoración que existe por asociar a un doctor en educación con un investigador, los decanos entrevistados reconocen que en sus facultades la investigación es un área que posee menos desarrollo, tradición y recursos que la docencia de pregrado, por tanto les resulta indisociable la figura del doctor en educación a la del docente universitario. De aquí que, a pesar de que se instala una demanda de doctores investigadores, la oferta laboral está asociada fundamentalmente a la docencia universitaria.

"Me interesa mucho que aporten en la docencia porque les da un vínculo muy concreto a la calidad del pregrado. Nuestro foco acá es un pregrado de calidad, no tenemos todavía una agenda de investigación bien desarrollada. Cada persona ha asumido un rol estratégico vinculado a la formación del pregrado y con dedicación en docencia. Eso les permite tener un cable a tierra muy vivo respecto a lo que significa el pregrado" (decano de una facultad de educación, universidad privada acreditada).

Por otra parte, el perfil ideal que se levanta desde las autoridades universitarias, alude a un académico especialista en los problemas y temas del sistema escolar. Se valora considerablemente que el doctor en educación posea vínculos concretos con el sistema educativo y que tenga herramientas para intervenir en él desde la investigación. Respecto a esta demanda, los decanos entrevistados consideran que los 
doctores en educación suelen distanciarse de la investigación aplicada o experimental. Les resulta difícil encontrar perfiles académicos que tengan experticia en intervención escolar, en investigación aplicada sobre modelos de enseñanza y aprendizaje y, muy especialmente, sobre didácticas de las distintas disciplinas escolares.

Sin duda se manifiesta una distancia evidente entre las demandas de algunos decanos y el perfil académico real de los doctores en educación que los mismos decanos declaran contratar en sus unidades académicas. Según los entrevistados, los doctores en educación egresados de programas nacionales poseen escasa experiencia investigativa, no han desarrollado experticia en la generación de proyectos y programas de investigación y trabajan muy débilmente con el sistema escolar. Las opiniones expresadas denotan una percepción crítica de la oferta nacional de posgrados y, también, agregan los entrevistados, de aquellos programas de formación semipresenciales o a distancia. Los sujetos consultados destacan la formación doctoral de aquellos programas que ponen el foco en la formación investigativa empírica y que enseñan a gestionar proyectos de investigación educativa.

No obstante lo anterior, las demandas más recurrentes que declaran los decanos entrevistados apuntan a valorar que un doctor en educación posea competencias transversales, como la capacidad de liderazgo, proactividad, empatía, inserción en equipos de trabajo y divulgación de sus ideas.

\section{b) La mirada de graduados y estudiantes de doctorados en educación}

Los otros actores entrevistados fueron graduados de programas doctorales en educación y estudiantes actuales de programas nacionales. Los temas analizados aluden a los criterios usados para decidir ingresar a un doctorado y a la percepción de los sujetos acerca de las fortalezas y debilidades de las propuestas formativas que conocieron o que están viviendo.

Entre las razones consideradas para elegir un programa de doctorado, los entrevistados aluden a argumentos vinculados a la 
factibilidad de cursarlo. Para ello, consideran que lo más relevante es la posibilidad de compatibilizar trabajo y estudio, y la modalidad de clases (presencial o semipresencial). Esta última razón es particularmente relevante en estudiantes que cursan doctorados que no están acreditados y que no pueden obtener becas. En un segundo orden de importancia, se señala el prestigio de la universidad, el vínculo con algún académico del programa, la presencia de menciones o líneas de investigación afines con sus intereses y la oportunidad de contar con otras experiencias y aprendizajes más allá del doctorado.

Al preguntarles con qué criterios escogerían hoy un programa de doctorado, la mayoría de los entrevistados puso el acento en la tradición y fortaleza que tenga el programa en el área de la investigación. Un grupo menor señaló que la calidad del programa y el acceso a becas fueron las razones principales en que basaron su decisión. La experiencia les permite reflexionar que lo central del doctorado es compartir con investigadores con mayor trayectoria, contar con acceso a investigadores actualizados y que se especialicen en las líneas o temas de interés del estudiante. Por lo mismo, les resulta central que al momento de elegir un doctorado se revisen las temáticas o ámbitos de especialización que ofrece y, al mismo tiempo, se analice la planta de académicos del programa para corroborar si se trata de profesionales que investigan, publican sus estudios y poseen líneas de investigación consolidadas. Por otra parte, también les parece relevante evaluar si el programa cuenta con acceso a bibliografía, buenas bases de datos, apoyo tecnológico y espacio físico adecuado para los doctorantes.

Los entrevistados pusieron énfasis en los procesos de admisión de un programa doctoral. Según ellos, la calidad de un programa comienza con el proceso de admisión. Al respecto, lo que más destacan como criterio de distinción y calidad de un programa es la rigurosidad para seleccionar a los postulantes. Ello implica contar con diversidad de instancias e instrumentos y, especialmente, con transparencia en la claridad de los criterios utilizados. Los graduados y estudiantes, especialmente de las instituciones nacionales, consideran que la mayor debilidad de la oferta curricular es la carencia de una vinculación temprana de los doctorantes con equipos y prácticas de investigación. 
"Lo que yo creo es que si quieren que uno aprenda a investigar tienen que vincularte a un grupo de investigación. No vas a aprender a investigar si no es investigando. Desde el principio el programa debe tener claro dónde va a vincular a ese estudiante de doctorado, pero el programa no está generando esa vinculación" (egresado de doctorado nacional acreditado).

Los entrevistados extrañan una formación más centrada en las lógicas de investigación y productividad académica y menos referida a las dinámicas de cursos teóricos o lectivos. Existe una percepción crítica acerca de la formación metodológica que reciben los estudiantes y graduados de las universidades de habla hispana. Los entrevistados insisten en que falta vincularse a equipos de investigación, a discusiones especializadas y a circuitos o contactos académicos que permitan formar parte de redes de estudios en educación. Consideran que los cursos de metodología cualitativa y cuantitativa son insuficientes para aprender a investigar, y que se requiere mayor orientación para aprender a formular proyectos, conseguir recursos, escribir y divulgar las investigaciones.

Otro punto crítico que parece relevante es la duración de la tesis. Los graduados de programas nacionales reconocen que no existen incentivos ("ni sistemas de presión", afirma un entrevistado) para terminar la tesis en un tiempo razonable. Por el contrario, el estudiante puede estar años vinculado a un programa y no terminar su trabajo de investigación.

Finalmente, dentro de los datos que nos parecen importantes, destaca la percepción de la mayoría de los entrevistados que, una vez en el mundo laboral, lo verdaderamente relevante es tener el grado de doctor, no la institución en la que se cursó el posgrado. Según ellos, las universidades que contratan doctores en educación en Chile no valoran suficientemente el prestigio del programa donde se cursó el doctorado.

Síntesis de las demandas de empleadores, graduados y estudiantes a los doctorados en educación: 
- Existe una percepción generalizada entre los decanos y autoridades universitarias entrevistadas de que los doctores en educación egresados de programas nacionales (como también de habla hispana) y especialmente aquellos de programas a distancia o semipresenciales, tienen una formación débil en lógicas de investigación y producción académica, como diseñar propuestas de investigación aplicada, postular y ganar proyectos de investigación en los concursos públicos, formar parte de redes de especialistas internacionales en una determinada materia y divulgar los resultados de los estudios a través de asistencias a congresos y publicaciones.

- Las autoridades de las facultades de educación demandan perfiles de doctores con competencias en la gestión de proyectos, en la docencia universitaria y que conozcan el sistema educativo. Además, aluden a competencias genéricas, como demostrar proactividad y capacidad de trabajar en equipo. Por otra parte, declaran que existe un déficit de especialistas en el área de las didácticas y en intervención educativa de escuelas en contexto de pobreza.

- La universidad, como institución, ha cambiado, por tanto han cambiando las demandas a los doctores que trabajan en universidades. Ya no se pide solamente a un doctor que posea una sólida formación en investigación, sino que también se esperan competencias en docencia, gestión de proyectos, publicación, además de competencias genéricas, como demostrar proactividad y capacidad de trabajar en equipo. Sin embargo, existe un cierto "círculo vicioso", pues mientras los encargados universitarios demandan perfiles que tengan competencias en investigación, reconocen que cuentan con pocos recursos para desarrollar la investigación en sus facultades y, a su vez, que la docencia universitaria es parte central del trabajo de un doctor en educación.

- Las entrevistas a estudiantes y graduados evidencian que sus exigencias académicas al momento de elegir un programa doctoral son bajas, a excepción de un grupo que accede a doctorados a través de becas del Estado. Para el grupo mayoritario, importa más elegir un programa que les permita financiar sus estudios y/o 
conciliar el trabajo con los estudios doctorales. Pero una vez que cursan el programa o que egresan de él, poseen más herramientas para distinguir la calidad del mismo. En este sentido, la mayoría de los entrevistados tiende a ser crítica con su formación de posgrado.

\section{Conclusiones}

En Chile, la oferta de formación de posgrado en educación sufre un gran vacío de regulación. Los resultados del estudio expuestos en este artículo, sobre el caso de los programas de doctorado en educación, sirven como un ejemplo para analizar esta problemática. Existe una oferta amplia y dispersa de programas doctorales que presentan objetivos de formación y núcleos curriculares variados, al igual que aproximaciones epistemológicas diversas sobre la investigación en educación. También, el análisis da cuenta de que estos programas otorgan distintos enfoques en la formación de investigadores (denominados "académico", "pragmático" y "experiencial"). Asimismo, se denotan distintos niveles de exigencia, tanto de entrada, como de egreso de los programas. En consecuencia, el título de doctor en educación no homologa estándares mínimos comunes para los perfiles de egresados.

Por otra parte, los actores vinculados con la oferta de posgrado (autoridades universitarias, egresados y estudiantes de programas doctorales), poseen una opinión crítica respecto a la calidad de la formación doctoral de los programas de educación nacionales. Dentro de las debilidades identificadas, destaca la baja presencia que tienen en las propuestas curriculares temas como la formación en investigación y la producción científica. Estas debilidades parecen más evidentes en el escenario académico actual, que demanda un perfil de doctor en educación que sepa demostrar capacidades en la elaboración proyectos, en la atracción de recursos de investigación y en la publicación de resultados de sus estudios en revistas científicas.

En este contexto, los procesos de acreditación resultan insuficientes, pues la mayoría de los estudiantes que cursan doctorados en educación, lo hacen en programas no acreditados. Si un 
programa no se acredita, puede, en términos reales, ofertar cualquier plan de estudio, pues no existen filtros de regulación públicos que permitan normar qué es lo mínimo y fundamental en la formación de doctores en educación. Esto confirma que los actuales procesos de acreditación de la educación superior chilena de posgrado pueden ser una contribución, pero que son insuficientes. Por lo anterior, es probable que algunas universidades no estén particularmente interesadas en acreditar sus programas de posgrado, pues existe un público creciente que demanda formación de este nivel, que opta por programas que le otorguen facilidades para compatibilizar el trabajo y el estudio, y que no necesariamente estén acreditadas, ni que puedan acceder a becas del Estado.

Junto al fortalecimiento de los procesos de regulación que normen las condiciones fundamentales con las que deben cumplir las universidades para ofertar programas de doctorado en educación, el Estado, además, podría contemplar incentivos estatales que promuevan la creación de programas doctorales de excelencia. Ello supone recursos, por cierto, pero condicionados a la generación de propuestas de alta calidad, con aseguramiento de líneas de investigación consolidadas, con convenios internacionales y una política de becas atractiva, que permita seleccionar postulantes de calidad. En esta perspectiva sería importante pensar en doctorados colaborativos en el ámbito nacional e internacional que permitan hacer sinergia entre los especialistas y áreas de investigación de distintas universidades. De esta manera, se rompería además con el aislamiento en el que trabajan las distintas universidades, y se promocionarían líneas de investigación que posean tradición y consistencia al interior de las instituciones.

Finalmente, es importante insistir en que los problemas que hemos reseñado para el caso del doctorado en educación en Chile deben ser entendidos en el contexto del escenario de fuerte desregulación de la educación superior chilena. En el campo de la educación, entonces, y muy particularmente en su nivel de posgrado, se requiere un poder regulador externo más presente, orientador y/o enmarcador de las ofertas posibles, si es que el objetivo final es aportar a consolidar una comunidad de investigadores educacionales de calidad. 


\section{Referencias bibliográficas}

Becker, G. (1983) Capital humano, Madrid: Alianza.

Bernasconi, A. y Rojas, A. (2004) Informe sobre la educación superior en Chile; 1980-2003, Santiago, Editorial Universitaria.

Brunner, J. J., Uribe, D. (2007) Mercados universitarias: los nuevos escenarios de la educación superior, Santiago: Universidad Diego Portales.

Collins, R. (1989) La sociedad credencialista, Madrid: Akal.

Dutercq, Y. (2005) La régulation des politiques déducation, Rennes, Presses: Universitaires de Rennes.

Jimeno Sacristán, J. y Pérez Gómez, A. (2008) Comprender y Transformar la Enseñanza, Madrid: Morata.

Krauskopf, M. (1999) Los doctorados en Chile: perfil y capacidad científica de los programas en ciencias acreditados en Chile, pp. 359-407) en Estudios Públicos 76, Santiago: CEP.

Maroy, C. (2004) Regulation and Inequalities in European Education Systems. Final Report, Université catholique de Louvain, GIRSEF, Louvain la Neuve.

Recibido: 27/09/2010

Aceptado: 24/04/2011 